\title{
1 Effects of contracture on gait kinematics: a systematic review
}

Michael Attias $^{\mathrm{a}, \mathrm{b}, \mathrm{d}}$; Odile Chevalley ${ }^{\mathrm{c}}$; Alice Bonnefoy-Mazure ${ }^{\mathrm{a}}$, Geraldo De Coulon $^{\mathrm{e}}$; Laurence Cheze $^{\mathrm{d}}$; Stéphane Armand ${ }^{\mathrm{a}}$

$\underline{\text { Institutions: }}$

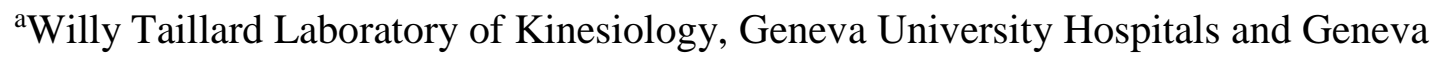
University, Switzerland.

${ }^{\mathrm{b}} \mathrm{HES}-\mathrm{SO}$ University of Applied Sciences and Arts Western Switzerland, School of Health Sciences, Geneva, Switzerland

${ }^{\mathrm{c} B e r n}$ University of Applied Sciences Bern, Switzerland

${ }^{\mathrm{d} U n i v e r s i t e ́ ~ C l a u d e ~ B e r n a r d ~ L y o n ~ 1, ~ V i l l e u r b a n n e, ~ F r a n c e ~}$

${ }^{\text {e}}$ Pediatric Orthopaedic Service, Department of Child and Adolescent, Geneva University Hospitals and Geneva University, Geneva, Switzerland.

\section{Corresponding author:}

E-mail: Michael.attias@hcuge.ch

Postal Address : Laboratoire de Cinésiologie Willy Taillard, rue Gabreille-Peret-Gentil 4, 1211 Genève 14 , Switzerland.

Phone number: +4122.372.78.27 / Fax: +41 (0)22 3727799

Word count for the abstract: 247

Word count for the main text: 2413

Number of Tables: 3

Number of Figures: 0 


\begin{abstract}
Background: Contractures of a major joint in the lower limbs may impair human walking in addition to other daily living activities. A contracture is defined as the inability of a joint to perform the full range of motion and excessive resistance during passive mobilization of the joint. Few studies have reported methods describing how to evaluate contractures. Understanding the association among all of these studies seems essential to improve patient management. Therefore, we conducted a systematic review on this topic to elucidate the influence of contractures on gait kinematics.
\end{abstract}

Methods: An electronic search in the literature will be conducted. Studies were screened by title and abstract and full texts were evaluated secondarily for definitive inclusion. The quality of the included studies was assessed independently by the two review authors with the Modified Quality Assessment Checklist. The included studies were separated into three categories: pathological contracture versus healthy controls (descriptive), simulated contracture versus healthy controls (experimental), and pre- and post-kinematics after surgical muscle lengthening (surgery).

Findings: From a total of 4402 references, 112 original articles were selected, and 28 studies were identified in this systematic review. No significant difference between raters was observed on the total score of the Modified Quality Assessment Checklist.

Interpretation: Contractures influence walking depending on the location (muscle) and the contracture level (muscle-tendon length). After giving a definition of contracture, this review identified some contracture alterations, such as plantarflexion, knee flexion and hip flexion contractures, with a kinematic description and presented possible different compensations.

Keywords: Systematic review, Contracture, Kinematics, Muscle-tendon length, Muscle shortening, Gait 


\section{Introduction}

A contracture is defined as the inability of a joint to perform the full range of motion and excessive resistance during passive mobilization of the joint (Gaudreault et al., 2009). The structures involved in contractures include ligaments, capsule, tendons and muscles for which the extensibility is limited and the stiffness is increased (Prabhu et al., 2013). Moreover, Perry and Burnfield (2010) differentiate between elastic and rigid contractures. An elastic contracture yields to the forceful stretch of an examiner or body weight, whereas a rigid contracture resists considerable force (stiffness) and is able to support body weight. Causes of contracture can be a result of different factors. Generally, a contracture is a common complication of many neurological and musculoskeletal conditions (Prabhu et al., 2013).

Contractures of a major joint in the lower limbs may impair human walking in addition to other daily living activities (Hoang et al., 2014; Prabhu et al., 2013).

Contractures have a high incidence in neurological and orthopedic conditions (Farmer and James, 2001), including ligament and joint capsule shortening, intra-articular adhesions, fibrofatty tissue proliferation into the joint and muscular shortening (Fox et al., 2000), muscle retraction, tendinous adherence, skin or subcutaneous tissue loss, joint capsule thickening or a combination of these factors (Roberson and Giurintano, 1995). The primary factors that cause suffering from contractures are burns, spinal cord injuries, cerebral palsy, strokes, and advanced age (Hoang et al., 2014). The association among contracture, muscle weakness and spasticity is generally accepted, although it has not been demonstrated scientifically (Hoang et al., 2014).

The main effect of contractures is a limitation of joint range of motion resulting from soft tissue stiffness (Hoang et al., 2014), but contractures can also cause pain, sleep disturbances, pressure ulcers, and deformities (Fox et al., 2000; Prabhu et al., 2013). A limited range of 
motion affects mobility and daily life activities. Among these activities, walking is a primordial activity of daily living. Patients and therapists ranked "moving around indoors" as the most important activity to preserve/restore in patients (Chiou and Burnett, 1985). Contractures induce gait deviations and limit a person's ability to walk (Hoang et al., 2014). In children with Duchenne muscular dystrophy, joint contracture represents the second most often occurring major clinical impairment that results in gait deviations (Gaudreault et al., 2009). In cerebral palsy, the commonest orthopedic deformity is equinus deformity caused by the contracture of the triceps surae (Galli et al., 2005). To choose the best therapeutic strategy in relation to gait deviations, it is mandatory to understand the gait deviations and to distinguish the primary deviation (directly resulting from the pathology) and the secondary deviation (compensatory mechanisms) (Schmid et al., 2013). Therefore, understanding the effects of contractures on gait deviations is important to support the therapeutic choice. In the literature, different approaches have been used to elucidate the effect of contractures on gait.

The first approach is to compare the gait patterns between persons with contractures and matched healthy persons (Gaudreault et al., 2009; Svehlik et al., 2010). The second approach is to replicate the gait patterns of a patient with contractures by having healthy participants imitate the gait patterns. To this end, the investigators ask the participants to replicate the gait pattern by imitating patients with contractures, e.g., to walk on their tiptoes to imitate a triceps contracture (Romkes and Brunner, 2007). The third approach is to replicate the gait patterns of a patient with contractures in healthy participants by induced constraints. In this case, the healthy participants are constrained in certain joint degrees of freedom with an exoskeleton (Matjacic and Olensek, 2007; Matjacic et al., 2006) or an orthosis (Houx et al., 2012; Houx et al., 2013). The fourth approach is to use a computer simulation to evaluate the effects of constraints induced by contractures on gait (Goldberg and Neptune, 2007; Hicks et al., 2008; Neptune et al., 2007). Finally, the fifth approach is based on the comparison of a patient's gait 
before and after treating the contracture (Baddar et al., 2002; Chimera et al., 2012; Galli et al., 2005, 2009; Hemo et al., 2006; Kadhim and Miller, 2014; Lofterod and Terjesen, 2008; Park et al., 2006; Tylkowski et al., 2009). These studies have demonstrated the importance of studying this subject. Associating these studies seems essential to improve patient management. Therefore, we conducted a systematic review on this topic to elucidate the influence of contractures on gait kinematics.

\section{Methods}

\subsection{Search strategy}

To provide a comprehensive overview of the literature, an electronic search will be conducted within the following databases: MEDLINE/PubMed, from January 1948 to July 2014; CINHAL (EBSCO), from January 1981 to July 2014; and EMBASE, from January 1980 to July 2014. In addition, the references of included studies will be searched to identify further studies. The search strategy will encompass the following search terms: contracture, muscle shortening, muscle length, range of motion, joint flexibility, kinematics, biomechanics, walking, gait, and locomotion. The search strategy for the databank MEDLINE will look as follows: \#1 (\#contracture OR \# contracture* OR \# muscle shortening OR \# muscle length* OR \# range of motion OR \# joint flexibility); \#2 (\#kinematics OR \# biomechanics); \#3 (\#walking OR \# gait OR \# locomotion); and \#4 (\#1 AND \#2 AND \#3).

\subsection{Inclusion and exclusion criteria}

Studies were screened by title and abstract following the rules of inclusion and exclusion criteria (Table 1). Full texts were evaluated secondarily for definitive inclusion. Two categories were selected to keep a maximum of articles, embracing concerned fields. The inclusion criteria contained articles about patient and healthy controls, as well as the simulation of contracture by different means. This review also included studies about muscle 
lengthening surgical intervention; these studies observed the pre- and post-outcome on gait kinematics. Two review authors independently screened the titles and abstracts. Disagreements were resolved by discussion until consensus, and when necessary, a third author arbitrated. Next, reviewers subsequently independently assessed the full-length reports for eligibility of the studies based on the inclusion and exclusion criteria. Disagreements were also discussed and arbitrated by a third author if necessary.

\subsection{Data extraction and quality assessment}

The two review authors independently extracted data from the included studies with a customized form. The following data were extracted: study design or aim, study population characteristics (diagnosis, age, sample size), parameters evaluated (kinematics, kinetics, EMG, etc.), major joints assessed and/or device, and main results (especially kinematics).

The quality of the included studies was assessed independently by the two review authors with the Modified Quality Assessment Checklist (Schmid et al., 2013). The checklist was first described by (Downs and Black, 1998). The authors developed this tool to assess the quality of randomized and non-randomized trials. It has a high internal consistency, good test-retest and inter-rater reliability, and good face and criterion validity. The checklist was modified to assess the quality of non-randomized observational studies (Schmid et al., 2013). The included studies are evaluated with 17 items with a total maximum score of 20 points. These 17 items were then classified in 5 categories and evaluated as follow: reporting quality (8 items, maximum 10 points), external validity ( 3 items, maximum 3 points), internal validity bias ( 3 items, maximum 3 points), internal validity - confounding ( 2 items, maximum 2 points), and statistical power (1 item, maximum 2 points).

\subsection{Data Analysis}


The extracted data were analyzed in a qualitative manner. The included studies were separated into three categories: pathological contracture versus healthy controls (descriptive), simulated contracture versus healthy controls (experimental), and pre- and post-kinematics after surgical muscle lengthening (surgery). Given that it is difficult to assess the contribution of a single surgery during a multi-level surgery (Lofterod and Terjesen, 2008), we investigated only single surgeries.

An intraclass correlation coefficient (ICC) with (+/- confidence interval) and the p-value (ttest) were calculated to evaluate the inter-rater reliability on the total score of the modified quality assessment. ICC was described according to Shrout and Fleiss (1979) (> 0.75 excellent reliability, $0.4-0.75$ fair to good reliability and $<0.4$ poor reliability). Statistical analyses were performed using MATLAB R2012b (MathWorks, USA).

\section{Results}

\subsection{Selection of studies}

A total of 4402 references were found after the electronic database research. After screening the titles and abstracts and removing duplicates, 112 original articles were selected. Following the request for inclusion and exclusion criteria, 28 studies were identified in this systematic review. The major part of the excluded articles was due: 1) multilevel surgeries (35), 2) absence or inaccurate explanation about contractures (33), 3) absence or incomplete kinematic data (8), 4) letter to editor or commentary (5), 5) abstract (2) and 6) literature review (1). These 28 included studies were then classified in three groups: experimental (15), descriptive (6) and surgery (7). In the experimental group, 9 articles focused on plantarflexion contracture, 5 on knee flexion contracture, and 1 on hip and knee flexion contracture. Ten studies focused on adults and 5 on children. Twelve used an exoskeleton or an orthosis to impose contractures, 1 used tapes and 2 imitated gait pattern of patients with contractures. In 
the descriptive group, 3 articles focused on plantarflexion contracture, 1 on stiff hip, 1 on hip flexion contracture and 1 on hip and knee flexion contracture. These 6 articles focused on children. In the surgery group, 7 articles focused on plantarflexion contracture (gastrocnemius, soleus or achille tendon lengthening) with 6 on children and 1 on adults. The extracted data from the included studies are reported in Table 2: study design or aim, number of subjects (gender and participants type), mean age in years, parameters evaluated, major joints assessed and/or device and main results.

\subsection{Quality assessment}

The Modified Quality Assessment Checklist with the evaluation for reporting quality, external validity, internal validity - bias, internal validity - confounding, and statistical power are described in Table 3. The ICC (+/- confidence interval) $0.87(0.94 / 0.73)$ on the total score of the modified quality assessment showed excellent reliability (Shrout and Fleiss, 1979) and a p-value of 0.783 between raters; no significant difference was observed. In the 28 studies, the total mean score and standard deviation (SD) of the modified quality assessment was computed and was $12.8(2.0)$ on 20 points. The mean score (SD) for reporting was 8.4 (1.3) on 10 points; for external validity, 1.3 (0.6) on 3 points; for internal validity (Bias), $2.0(0.8)$ on 3 points; for internal validity (confounding), 1 (0.6) on 2 points, and for power, $0.1(0.4)$ on 2 points.

\section{Discussion}

This systematic review aims to characterize the influence of contractures on gait kinematics. Twenty-eight articles were selected and evaluated by 2 reviewers with excellent inter-rater reliability. These articles were divided in three groups according to the study method of the contractures (experimental, descriptive and surgery studies). Even if the definition of contracture is not consistent in all the studies, they agree that a contracture induces a restricted passive range of motion of one or several joints. It also frequently returned that contractures 
appear as a result of pathologic conditions (such as burns, spinal cord injury, cerebral palsy, stroke, elderly age (Hoang et al., 2014), and impaired walking).

The primary reported contractures that have an effect on gait are ankle plantarflexion (gastrocnemius or soleus contracture), knee flexion (hamstrings contracture) and hip flexion (psoas contracture).

A plantar flexion contracture can be the result of gastrocnemius muscle retraction or soleus muscle retraction if it is not a neurological problem. Several authors have studied walking in plantarflexion without separating the effect of gastrocnemius and soleus contractures. These studies mainly showed a loss of heel strike at initial contact (loss of first ankle rocker), ankle plantarflexion (instead of dorsiflexion) occurring at midstance, and disruption of the third rocker in a terminal stance (Davids et al., 1999). Compensations showed a limitation in both hip and knee extension (stance phase) without a short hamstring or hip flexor (Goodman et al., 2004), but another study showed an increase in knee extension during midstance and specify that some subjects walk in knee flexion (Leung et al., 2014). Therefore, the different compensations occur at the knee level with a plantarflexion contracture: knee flexion or knee extension during mid-stance. An increase of pelvic tilt, an increase of knee varus, reduction of hip adduction, and more internal foot progression were also reported (Houx et al., 2013). The minimal plantarflexion contracture to significantly change kinematics and kinetics is $10^{\circ}$ of plantarflexion (Houx et al., 2012).

Knee flexion contracture has an effect on excessive knee flexion during walking commonly observed in a crouch gait (Balzer et al., 2013). Compensations that have been observed include a decreased stride length and velocity, increased forefoot weight bearing and flexion posture in stance (Cerny et al., 1994). Matjacic and Olensek (2007) described knee flexion contracture by hamstring contracture. The minimal popliteal angle (hamstring contracture) to 
have an effect on gait was found to be $85^{\circ}$ and included increased walking effort, decreased speed, decreased stride and step length and increased knee flexion in stance (Whitehead et al., 2007).

Compensations of a knee flexion contracture include increased maximal dorsiflexion, eversion and external rotation of the hindfoot (Balzer et al., 2013), decreased hip flexion in stance, increased posterior pelvic tilt, decreased pelvic obliquity and rotation and premature ankle dorsi and plantarflexion in stance (Whitehead et al., 2007). It seems that we need a minimum of $30^{\circ}$ of knee flexion contracture to change trunk kinematics in all planes. In the coronal plane, the trunk is tilted to the contracture side in standing and walking. In the sagittal plane, anterior inclination of the trunk increased during walking. In the axial plane, trunk rotation to the unaffected side decreased during walking (Harato et al., 2008a, b). Knee flexion contracture by hamstring contracture, when it is associated with iliopsoas contracture, leads to a tendency to dorsiflex the ankle angle during midstance while the hamstring only shifted the hip angle toward extension (Matjacic and Olensek, 2007). When we analyze knee flexion during walking, we need to be careful not to systematically associate this flexion with a contracture because $80 \%$ of subjects with a crouch gait for example, had a hamstring with a normal length or longer despite persistent knee flexion during stance; it seems that this was a problem with hip flexion with a psoas contracture (Delp et al., 1996).

Regarding a hip flexion contracture (psoas contracture), Matjacic and Olensek (2007) described a hip flexion because of a psoas contracture linked with knee flexion during stance. An ankle dorsiflexion during midstance was observed in association with a hamstring contracture, as was described above. All subjects with a crouch gait had a psoas during walking that was shorter than normal by more than 1 standard deviation (Delp et al., 1996). 
Numerous studies have examined a multilevel contracture because it is difficult to have subjects with only one contracture. Moreover, a contracture is often associated with other alterations, such as muscle weakness. The physiopathology of contractures and how they develop remains unknown.

\section{Conclusions}

Contractures influence walking depending on the location (muscle) and the contracture level (muscle-tendon length). Different types of research studied contractures in experimental, descriptive and surgery studies. After establishing a definition for contracture, this review identified several contracture alterations, such as plantarflexion, knee flexion and hip flexion contractures, with a kinematic description and presented possible different compensations. By continuing to study and to differentiate gait alterations from compensations, treatment can be better targeted (e.g., surgery and physiotherapy).

\section{Acknowledgments}

This study was founded by the Swiss National Science Foundation (SNF) project 325230_146801. 


\section{References}

Baddar, A., Granata, K., Damiano, D.L., Carmines, D.V., Blanco, J.S., Abel, M.F., 2002. Ankle and knee coupling in patients with spastic diplegia: effects of gastrocnemius-soleus lengthening. Journal of Bone \& Joint Surgery, American Volume 84A, 736-744.

Balzer, J., Schelldorfer, S., Bauer, C., van der Linden, M.L., 2013. Effects of simulated crouch gait on foot kinematics and kinetics in healthy children. Gait \& posture 38, 619-624.

Cerny, K., Perry, J., Walker, J.M., 1994. Adaptations during the stance phase of gait for simulated flexion contractures at the knee. Orthopedics 17, 501-513.

Chimera, N.J., Castro, M., Davis, I., Manal, K., 2012. The effect of isolated gastrocnemius contracture and gastrocnemius recession on lower extremity kinematics and kinetics during stance. Clinical Biomechanics 27, 917-923.

Chiou, II, Burnett, C.N., 1985. Values of activities of daily living. A survey of stroke patients and their home therapists. Phys Ther 65, 901-906.

Davids, J.R., Foti, T., Dabelstein, J., Bagley, A., 1999. Voluntary (normal) versus obligatory (cerebral palsy) toe-walking in children: a kinematic, kinetic, and electromyographic analysis. Journal of pediatric orthopedics 19, 461-469.

Delp, S.L., Arnold, A.S., Speers, R.A., Moore, C.A., 1996. Hamstrings and psoas lengths during normal and crouch gait: Implications for muscle-tendon surgery. Journal of Orthopaedic Research 14, 144-151.

Downs, S.H., Black, N., 1998. The feasibility of creating a checklist for the assessment of the methodological quality both of randomised and non-randomised studies of health care interventions. Journal of epidemiology and community health 52, 377-384.

Farmer, S.E., James, M., 2001. Contractures in orthopaedic and neurological conditions: a review of causes and treatment. Disabil Rehabil 23, 549-558.

Fox, P., Richardson, J., McInnes, B., Tait, D., Bedard, M., 2000. Effectiveness of a bed positioning program for treating older adults with knee contractures who are institutionalized. Phys Ther 80, 363-372.

Galli, M., Cimolin, V., Crivellini, M., Albertini, G., 2005. Gait analysis before and after gastrocnemius fascia lengthening in children with cerebral palsy. Journal of Applied Biomaterials and Biomechanics 3, 98-105.

Galli, M., Cimolin, V., Crivellini, M., Albertini, G., 2009. Long-term evaluation of isolated gastrocnemius fascia lengthening in children with cerebral palsy using gait analysis. Journal of Pediatric Orthopaedics: Part B 18, 228-233.

Gaudreault, N., Gravel, D., Nadeau, S., 2009. Evaluation of plantar flexion contracture contribution during the gait of children with Duchenne muscular dystrophy. Journal of Electromyography and Kinesiology 19, e180-e186.

Goldberg, E.J., Neptune, R.R., 2007. Compensatory strategies during normal walking in response to muscle weakness and increased hip joint stiffness. Gait \& posture 25, 360-367.

Goodman, M.J., Menown, J.L., West, J.M., Jr., Barr, K.M., Vander Linden, D.W., McMulkin, M.L., 2004. Secondary gait compensations in individuals without neuromuscular involvement following a unilateral imposed equinus constraint. Gait \& posture 20, 238-244.

Harato, K., Nagura, T., Matsumoto, H., Otani, T., Toyama, Y., Suda, Y., 2008a. A gait analysis of simulated knee flexion contracture to elucidate knee-spine syndrome. Gait and Posture 28, 687-692.

Harato, K., Nagura, T., Matsumoto, H., Otani, T., Toyama, Y., Suda, Y., 2008b. Knee flexion contracture will lead to mechanical overload in both limbs: A simulation study using gait analysis. Knee 15, 467-472. 
Hemo, Y., Macdessi, S.J., Pierce, R.A., Aiona, M.D., Sussman, M.D., 2006. Outcome of patients after achilles tendon lengthening for treatment of idiopathic toe walking. Journal of Pediatric Orthopaedics 26, 336-340.

Hicks, J.L., Schwartz, M.H., Arnold, A.S., Delp, S.L., 2008. Crouched postures reduce the capacity of muscles to extend the hip and knee during the single-limb stance phase of gait. Journal of biomechanics 41, 960-967.

Hoang, P.D., Gandevia, S.C., Herbert, R.D., 2014. Prevalence of joint contractures and muscle weakness in people with multiple sclerosis. Disabil Rehabil 36, 1588-1593.

Houx, L., Brochard, S., Lempereur, M., Remy-Neris, O., 2012. Simulation of unilateral equinus using an adjustable orthosis in children: design, feasibility and biomechanical effects.

Prosthetics \& Orthotics International (Taylor \& Francis Ltd) 36, 131-136.

Houx, L., Lempereur, M., Remy-Neris, O., Brochard, S., 2013. Threshold of equinus which alters biomechanical gait parameters in children. Gait and Posture 38, 582-589.

Kadhim, M., Miller, F., 2014. Crouch gait changes after planovalgus foot deformity correction in ambulatory children with cerebral palsy. Gait and Posture 39, 793-798.

Leung, J., Smith, R., Harvey, L.A., Moseley, A.M., Chapparo, J., 2014. The impact of simulated ankle plantarflexion contracture on the knee joint during stance phase of gait: A within-subject study. Clinical Biomechanics 29, 423-428.

Lofterod, B., Terjesen, T., 2008. Local and distant effects of isolated calf muscle lengthening in children with cerebral palsy and equinus gait. Journal of children's orthopaedics 2, 55-61.

Matjacic, Z., Olensek, A., 2007. Biomechanical characterization and clinical implications of artificially induced crouch walking: Differences between pure iliopsoas, pure hamstrings and combination of iliopsoas and hamstrings contractures. Journal of biomechanics 40, 491-501.

Matjacic, Z., Olensek, A., Bajd, T., 2006. Biomechanical characterization and clinical implications of artificially induced toe-walking: Differences between pure soleus, pure gastrocnemius and combination of soleus and gastrocnemius contractures. Journal of biomechanics 39, 255-266.

Neptune, R.R., Burnfield, J.M., Mulroy, S.J., 2007. The neuromuscular demands of toe walking: A forward dynamics simulation analysis. Journal of biomechanics 40, 1293-1300.

Park, C.I., Park, E.S., Kim, H.W., Rha, D.W., 2006. Soft tissue surgery for equinus deformity in spastic hemiplegic cerebral palsy: Effects on kinematic and kinetic parameters. Yonsei medical journal 47, 657-666.

Perry, J., Burnfield, J., 2010. Gait Analysis: Normal and Pathological Function,. Slack, Pomona, CA.

Prabhu, R.K., Swaminathan, N., Harvey, L.A., 2013. Passive movements for the treatment and prevention of contractures. The Cochrane database of systematic reviews 12, CD009331.

Roberson, L., Giurintano, D.J., 1995. Objective measures of joint stiffness. Journal of hand therapy : official journal of the American Society of Hand Therapists 8, 163-166.

Romkes, J., Brunner, R., 2007. An electromyographic analysis of obligatory (hemiplegic cerebral palsy) and voluntary (normal) unilateral toe-walking. Gait \& posture 26, 577-586.

Schmid, S., Schweizer, K., Romkes, J., Lorenzetti, S., Brunner, R., 2013. Secondary gait deviations in patients with and without neurological involvement: a systematic review. Gait \& posture 37, 480-493.

Shrout, P.E., Fleiss, J.L., 1979. Intraclass correlations: uses in assessing rater reliability. Psychological bulletin 86, 420-428.

Svehlik, M., Zwick, E.B., Steinwender, G., Saraph, V., Linhart, W.E., 2010. Genu recurvatum in cerebral palsy - Part A: Influence of dynamic and fixed equinus deformity on the timing of knee recurvatum in children with cerebral palsy. Journal of Pediatric Orthopaedics Part B 19, 366-372. 
Tylkowski, C.M., Horan, M., Oeffinger, D.J., 2009. Outcomes of Gastrocnemius-soleus Complex Lengthening for Isolated Equinus Contracture in Children With Cerebral Palsy. Journal of pediatric orthopedics 29, 771-778.

Whitehead, C.L., Hillman, S.J., Richardson, A.M., Hazlewood, M.E., Robb, J.E., 2007. The effect of simulated hamstring shortening on gait in normal subjects. Gait \& posture 26, 90-96. 
Table 1 : Inclusion and exclusion criteria

\begin{tabular}{|c|c|c|}
\hline & Inclusion & Exclusion \\
\hline Population & $\begin{array}{l}\text { Contractures or simulated } \\
\text { contractures and healthy } \\
\text { controls. } \\
\text { Pre-intervention (muscle } \\
\text { lengthening) versus post- } \\
\text { intervention }\end{array}$ & $\begin{array}{l}\text { Acute contracture, sportsmen, } \\
\text { animal } \\
\text { Splint as treatment, or splint } \\
\text { with pathology }\end{array}$ \\
\hline Intervention & $\begin{array}{l}\text { Observation, evaluation, } \\
\text { interpretation, Clinical Gait } \\
\text { Analysis }\end{array}$ & $\begin{array}{l}\text { Cast, splint, stretching, } \\
\text { botulinum toxin, multi- surgery }\end{array}$ \\
\hline Outcome & $\begin{array}{l}\text { Kinematics, gait deviations, } \\
\text { compensations }\end{array}$ & No kinematics \\
\hline$T_{\text {ype of study }}$ & $\begin{array}{l}\text { Original research, published in } \\
\text { peer-reviewed scientific } \\
\text { journals, case-study research } \\
\text { (observational) }\end{array}$ & $\begin{array}{l}\text { Conference abstracts, non peer- } \\
\text { reviewed publications, single } \\
\text { case, systematic review. }\end{array}$ \\
\hline
\end{tabular}


Table 2: Extracted data from the included studies

First author

Study design or aim

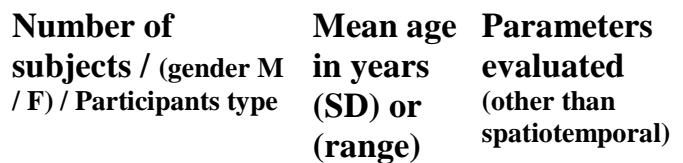

Major joints assessed and/or device

\section{Experimentation}

Balzer

2013

Cerny

1994

Davids Evaluate primary from secondary 1999

Goodman 2004

Harato 2008a

Investigate static and dynamic changes in trunk kinematics with simulated knee flexion contracture during walking. bilateral knee flexion contracture (specially designed hip-knee-belt, to contracture of $40^{\circ}$ ) causes compensatory mechanisms in foot motion during gait in healthy children.

Adaptation in the stance phase of gait to knee flexion contractures simulated by a knee-ankle-foot orthosis. compensatory with normal children, walking normally and voluntarily toegait pattern.

Determine what compensatory gait deviations may occur as a result of an imposed, unilateral equinus constraint. produce a symmetric artificial knee flexion walking in comparison to real toe walking

Harato Investigate the effect of knee flexion 2008b contracture on the knee mechanics both in affected and contralateral limbs during gait.

Houx 2012 Understand consequences of equinus foot on gait. Development of orthosis to induce equinus.
30 healthy children

20 healthy women

$10.6(+/-$

Kinematics

knee flexion

Kinetics contracture

Kinematic
12 normal adult subjects

10 healthy females

$62(60-64)$

32 normal children; 9.3 (5.4-

15 toe walker

$13.6) ; 8.2$

EMG

Kinetics, EMG

\section{Kinetics \\ Kinematics}

23.7 (SD)

Kinematics Kinetics

Imposed ankle equinus by tape
Imitation of plantarflexion (toewalking)
Knee flexion contracture 
Houx 2013 Define threshold angle of equinus beyond

Leung Describe the effect of simulated 2014 plantarflexion contractures on knee on the spatiotemporal characteristics of

Matjacic Characterize biomechanically three 2006

Matjacic 2007

Olensek Evaluate and compare gait variability as 2005 recorded in normal gait and when being constrained by a mechanical exoskeleton with elastic ropes.

Ota 2014 Investigate the acute influence of varied levels of restricted ankle dorsiflexion on knee joint sagittal and frontal plane kinematics and kinetics during gait using a dial lock joint and allowing unrestricted plantar flexion which significant changes in 3D lower limb kinematics and kinetics occur in typically developing children biomechanics during the stance phase and gait by an ankle-foot-orthosis

10 healthy children

$9.7(8-12)$ different toe-walking gait patterns, artificially induced, by a mechanical exoskeleton with elastic ropes, in six neurologically intact subjects and to compare them to selected cases of pathological toe-walking.

\begin{tabular}{|c|c|c|}
\hline $\begin{array}{l}13 \text { able-bodied } \\
\text { adults }\end{array}$ & $30(+/-7)$ & $\begin{array}{l}\text { Kinematics } \\
\text { Kinetics }\end{array}$ \\
\hline
\end{tabular}

6 neurologically intact subjects

males
22.5 (+/-1.9) Kinematics Kinetics

Kinematics

Kinetics

Induced an adjustabl degree of unilateral equinus
Emulated contractures of soleu muscles and gastrocnemius
8 neurologically intact subjects males

\author{
$25.2(+/-3.5) \quad$ Kinematics \\ Kinetics
}

Emulated contractures of iliopsoas and hamstrings muscles by an exoskeleton with artificial muscles

\section{6 neurologically intact subjects males}

\section{5 (+/-1.9) Kinematics Kinetics}

Emulated contractures of soleu and gastrocnemius muscles
30 (15 / 15) healthy volunteers
21.7 (+/-2.2) Kinematics Kinetics

Restricted ankle dorsiflexion 
Romkes Understand the differences between 2007 obligatory toe-walking as observed in hemiplegic $\mathrm{CP}$ gait and voluntary toewalking.

Whitehead Determine the effect of simulated 2006 hamstring shortening on gait in normal subjects by an adjustable brace.
$12(8 / 4)$

hemiplegic

children cerebral

palsy $(\mathrm{CP}) ; 10$

healthy adult (5 / 5)

6 normal female volunteers

$26.2(21-42) \quad$ Kinematics

Kinetics,

Physiologica

1 cost index
Simulated hamstring shortening
Healthy subjects mimicking toewalking compared to hemiplegic $\mathrm{CP}$. $6.9(+/-\quad$ Kinematics

1.6) ; 7.6 Kinetics $(+/-2.4)$
Investigate the clinical relevance of hip 24 cerebral palsy kinematic and kinetic parameters, and 3D children ; 28 modelled psoas length in terms of discriminant validity, convergent validity, and responsiveness normal children

14 subjects with crouch gait ; 10 subjects without movement disorders
10.5 12.6
Kinematics and psoas during normal and crouch gait on the basis of a computer model of the lower limb. Compare the lenghts of subjects'hamstrings and psoas muscle during walking with their lengths during static muscles tests.

Gaudreault 2009

Quantify the passive moments of force produced by ankle plantar flexor contractures and determine their mechanical contribution to the net ankle moment calculated during the gait of children with Duchenne muscular dystrophy (DMD).

Gordon 2013 Describe the quantitative gait findings of the adolescent subject with a unilateral stiff hip and Determine whether these findings are similar to those of subjects presenting after arthrodesis.

\section{1 children with $9.2(+/-\quad$ Kinematics DMD; 14 healthy 2.6);9.7 Kinetics \\ Plantar flexion contractures} control children (+/-1.9)

6 children with stiff $13-17$ hip

Kinematics

Stiff hip
Hip flexor (psoas) contracture

Hamstrings and psoas lengths 
Svehlik

2010a

Svehlik

2010b
Detect outcome measures that could help differentiate between dynamic equinus and fixed equinus deformities. Describe the function of the gastrocnemius and soleus muscles when either dynamic triceps surae tightness or fixed equinus contracture was present.

Confirm the hypothesis of the influence of the dynamic and fixed equinus deformity on the timing of knee recurvation (hyperextension).
$23 \mathrm{CP}$ children ; none Kinematics $12 \mathrm{C}$ surae tightness and fixed equinus contracture

\author{
E-REC Kinematics \\ $8.01(+/-\quad$ Kinetics \\ 2.7) EMG \\ L-REC \\ $7.62(+/-$ \\ 2.36) \\ Healthy \\ Children \\ $10.32(+/-$ \\ 2.96)
}

late recurvatum excursion of the gastrocnemius-soleus muscle-tendon unit in relation to knee and ankle kinematics.

Chimera 2012

Galli 2005

Galli 2009
Compare the sagittal plane ankle and knee mechanics during the stance phase of gait in patients with non-spastic isolated gastrocnemius contracture (IGC) associated with overuse foot pathology to those of healthy controls. Determine if sagittal plane ankle and knee mechanics would be altered with gastrocnemius recession. Compare post-surgical ankle and knee sagittal plane mechanics in these patients to those of healthy control participants.

Analyze kinematic and kinetic effects of gastrocnemius fascia lengthening on gait pattern in $\mathrm{CP}$ children and the evaluation of push-off ability before and after treatment.

Analyze kinematic and kinetic effects of gastrocnemius fascia lengthening over time using gait analysis in cerebral palsy (CP)
$34(17 / 17) \mathrm{CP}$ children 78 normal children

\author{
$6(1 / 5)$ adult \\ patients clinically \\ diagnosed with \\ 53.2 (+/- Kinematics \\ 5) ; $48.9 \quad$ Kinetics \\ non-spastic IGC ; \\ 33 (15 / 18) healthy \\ control participants
}

$7.2(+/-$

$3.4) ; 6.9$

Kinematics

$(+/-2.8)$

EMG
20 CP children with equinus 10 healthy children

$12 \mathrm{CP}$ children with equinus 20 healthy children
$8(+/-2.7) \quad$ Kinematics ; $9+/-3.1 \quad$ Kinetics

$9.3(+/-$ $2.7) ; 9$ $(+/-3.1)$
Kinematics Kinetics
Equinus gait and knee extension

\author{
Dynamic and fixed \\ equinus deformity on \\ the timing of knee \\ recurvation \\ (hyperextension)
}

Isolated gastrocnemius contracture with full knee extension
Gastrocnemius fascia lengthening and the effects at the knee joint

Gastrocnemius fascia lengthening and the effects at the knee joint 
Hemo 2006 Determine whether children maintain adequate power generation at the ankle joint and how the kinematic parameters change after Achilles tendon lengthening for treatment of idiopathic toe walking (ITW)
Lofterod 2008
Assess the local and distant effects of isolated calf muscle lengthening in ambulant $\mathrm{CP}$ children.

\section{ITW children}

$15(8 / 7) \mathrm{CP}$

children

27 CP children

Evaluated the outcomes of operative

2009 intervention for isolated equinus

contractures in individuals with $\mathrm{CP}$ using instrumented gait analysis.
9 (4.2- $\quad$ Kinematics

13.1) Kinetics

8.8 (6-14) Kinematics Kinetics

$11.4(+/-\quad$ Kinematics

3.2) Kinetics
Achilles tendon lengthening for treatment of idiopathic toe walking

Equinus gait with isolated calf muscle lengthening
Gastrocnemiussoleus complex lengthenings on isolated equinus contracture 
Table 3 : Modified Quality Assessment evaluated by MA and OC.

$\begin{array}{lllcccc}\text { Study ID } & \text { Reporting } & \begin{array}{c}\text { External } \\ \text { validity }\end{array} & \begin{array}{c}\text { Internal } \\ \text { validity } \\ \text { (Bias) }\end{array} & \begin{array}{c}\text { Internal } \\ \text { validity } \\ \text { (Confounding) }\end{array} & \text { Power } & \text { Total } \\ & & & & \end{array}$

\begin{tabular}{llllllllllll}
\hline MA & OC & MA & OC & MA & OC & MA & OC & MA & OC & MA & OC \\
\hline
\end{tabular}

\section{Experimentation}

Balzer 2013

Cerny 1994

Davids 1999

Goodman 2004

Harato 2008a

Harato 2008b

Houx 2012

Houx 2013

Leung 2014

Matjacic 2006

Matjacic 2007

Olensek 2005

Ota 2014

Romkes 2007

$\begin{array}{rrrrrrrrrrrr}10 & 10 & 1 & 1 & 3 & 3 & 0 & 1 & 0 & 0 & 14 & 15 \\ 9 & 8 & 1 & 1 & 2 & 2 & 1 & 2 & 0 & 0 & 13 & 13 \\ 9 & 8 & 2 & 1 & 3 & 3 & 1 & 2 & 0 & 0 & 15 & 14 \\ 8 & 6 & 1 & 0 & 1 & 1 & 1 & 2 & 0 & 0 & 11 & 9 \\ 7 & 9 & 1 & 1 & 3 & 2 & 0 & 1 & 0 & 0 & 11 & 13 \\ 9 & 9 & 1 & 1 & 3 & 2 & 1 & 2 & 0 & 0 & 14 & 14 \\ 9 & 9 & 1 & 1 & 3 & 3 & 1 & 2 & 0 & 0 & 14 & 15 \\ 9 & 9 & 1 & 1 & 3 & 3 & 1 & 2 & 0 & 0 & 14 & 15 \\ 9 & 8 & 1 & 1 & 3 & 2 & 1 & 2 & 0 & 0 & 14 & 13 \\ 11 & 9 & 1 & 1 & 1 & 1 & 1 & 2 & 0 & 0 & 14 & 13 \\ 11 & 9 & 1 & 1 & 1 & 1 & 1 & 2 & 0 & 0 & 14 & 13 \\ 10 & 9 & 1 & 1 & 1 & 1 & 1 & 2 & 0 & 0 & 13 & 13 \\ 9 & 9 & 1 & 2 & 3 & 2 & 1 & 2 & 0 & 0 & 14 & 15 \\ 8 & 8 & 2 & 1 & 2 & 2 & 0 & 1 & 0 & 0 & 12 & 12 \\ 7 & 9 & 1 & 0 & 1 & 1 & 0 & 1 & 0 & 0 & 9 & 11\end{array}$

\section{Description}

Choi 2011

Delp 1996

Gaudreault 2009

Gordon 2013

Svehlik 2010a

Svehlik 2010b

$\begin{array}{rrrrrrrrrrrr}9 & 9 & 2 & 2 & 2 & 3 & 0 & 1 & 2 & 2 & 15 & 17 \\ 7 & 7 & 2 & 2 & 1 & 2 & 1 & 2 & 0 & 0 & 11 & 13 \\ 9 & 8 & 2 & 2 & 3 & 3 & 1 & 1 & 0 & 0 & 15 & 14 \\ 5 & 4 & 2 & 3 & 1 & 0 & 0 & 1 & 0 & 0 & 8 & 8 \\ 9 & 8 & 2 & 1 & 2 & 1 & 0 & 2 & 0 & 0 & 13 & 12 \\ 9 & 9 & 2 & 1 & 2 & 2 & 1 & 1 & 0 & 0 & 14 & 13\end{array}$

\section{Surgery}

Baddar 2002

Chimera 2012

Galli 2005

Galli 2009

Hemo 2006

Lofterod 2008

\begin{tabular}{rrrrrrrrrrrr}
9 & 6 & 2 & 2 & 2 & 2 & 0 & 1 & 0 & 0 & 13 & 11 \\
10 & 10 & 2 & 1 & 2 & 3 & 1 & 2 & 0 & 0 & 15 & 16 \\
8 & 8 & 1 & 0 & 2 & 2 & 0 & 0 & 0 & 0 & 11 & 10 \\
8 & 8 & 1 & 0 & 2 & 2 & 0 & 0 & 0 & 0 & 11 & 10 \\
8 & 6 & 2 & 2 & 2 & 1 & 1 & 2 & 0 & 0 & 13 & 11 \\
8 & 7 & 2 & 1 & 2 & 1 & 0 & 1 & 0 & 0 & 12 & 10 \\
9 & 8 & 2 & 3 & 2 & 3 & 0 & 1 & 0 & 0 & 13 & 15 \\
\hline
\end{tabular}

Tylkowski 2009 